\title{
Molecular endpoints for establishing target engagement by novel idiopathic pulmonary fibrosis therapies
}

\author{
Paul J. Wolters ${ }^{1}$ and Vincent Cottin (10 ${ }^{2}$ \\ Affiliations: ${ }^{1}$ Division of Pulmonary, Critical Care, Allergy and Sleep Medicine, Dept of Medicine, University of \\ California, San Francisco, San Francisco, CA, USA. ${ }^{2}$ Respiratory Diseases Dept, National Reference Center for \\ Rare Pulmonary Diseases, Louis Pradel Hospital, Claude Bernard Lyon 1 University, Lyon, France.
}

Correspondence: Paul J. Wolters, University of California, San Francisco, Box 0111, San Francisco, CA 94143-0111, USA. E-mail: paul.woltersQucsf.edu

@ERSpublications

Measuring molecular endpoints in phase 2 clinical trials of IPF may be useful for designing subsequent phase 3 clinical trials http://ow.ly/li8J30nQUJx

Cite this article as: Wolters PJ, Cottin V. Molecular endpoints for establishing target engagement by novel idiopathic pulmonary fibrosis therapies. Eur Respir J 2019; 53: 1900283 [https://doi.org/10.1183/ 13993003.00283-2019].

Idiopathic pulmonary fibrosis (IPF) is a disease of progressive lung remodelling characterised by metaplastic epithelial cells, re-epithelialised air spaces (microscopic honeycombing), lymphoid aggregates, leukocyte accumulation (including macrophages, dendritic cells and mast cells), angiogenesis, lymphangiogenesis, fibroblast foci and excess matrix deposition [1,2]. On average, untreated patients diagnosed with IPF succumb to the disease within 3 years of diagnosis [2]. This dismal prognosis has improved following approval of pirfenidone and nintedanib for disease management $[3,4]$. These drugs cut disease progression in half, and likely improve survival. By showing that IPF is a treatable condition, approval of pirfenidone and nintedanib has accelerated interest in developing other medications that effectively treat IPF, with several nearing phase 3 of development [5].

Pirfenidone and nintedanib were approved in an era when no medications were available for treating IPF. This mandated that the medications be studied in placebo-controlled trials. With their success, it may prove challenging and ethically questionable to enrol large placebo-controlled clinical trials of novel IPF therapies using clinical measures as the primary endpoint, because patients may prefer to use pirfenidone or nintedanib as background therapy to ensure that, at a minimum, their disease progression is slowed. Placebo-controlled trials designed as add-on therapy on top of approved anti-fibrotic therapy will require large populations of patients [6]; therefore, increasing attention has been paid to preclinical and early clinical development. The alternative approach would be to enrol a placebo-controlled trial consisting of patients that have either failed or are intolerant to pirfenidone and nintedanib. Although this study design has been employed for small phase 2 trials $[7,8]$, it may be risky to propose such an approach for larger trials, because it remains uncertain whether there are sufficient numbers of patients failing with conventional therapy to enable enrolment in a reasonable timeframe. How these challenges with study design will play out in future studies remains to be seen, as a phase 3 trial testing a novel IPF therapy since the approval of pirfenidone and nintedanib has yet to be completed. In an ongoing phase 3 trial evaluating the efficacy and safety of GLPG1690 (Clinicaltrials.gov, NCT03733444 and NCT03711162), 
background therapy is the local standard of care for the treatment of IPF defined as either pirfenidone or nintedanib, or neither pirfenidone nor nintedanib (for any reason).

Considering these challenges, the study by KHaLil et al. [9] published in this issue of the European Respiratory Journal, investigated the safety, efficacy and pharmacokinetics of PBI-4050, a novel orally active small molecule, was evaluated. Pharmacokinetic profiles for PBI-4050 were similar in the PBI-4050 alone and PBI-4050+nintedanib groups but significantly reduced in the PBI-4050+pirfenidone group (reduced half-life of PBI-4050), suggesting a drug-drug interaction. Such interaction illustrates that the use of background therapy with pirfenidone or nintedanib may increase the complexity of drug development, and that pharmacokinetics and interaction need be carefully assessed before engaging in larger, placebo-controlled trials. Nevertheless, this 12-week open-label study did not find safety concerns with PBI-4050 and paved the way for its further development.

This study further illustrates additional barriers to designing future clinical trials, which include that the precise mechanism of action of PBI-4050 remains unknown, although the compound is known to bind the GPR40 and GPR84 G-protein coupled receptors. Similarly, the binding partners of pirfenidone and the most relevant kinase(s) inhibited by nintedanib remain unknown [10]. Furthermore, despite recent efforts [11-13], the cellular and molecular elements of lung remodelling that are modulated by these drugs remain unknown. Given the involvement of multiple pathways in the pathogenesis of IPF, it is conceivable that drugs targeting multiple molecules or pathways be more effective than more selective therapies [14]. However, not knowing specifically how pirfenidone or nintedanib slow IPF progression limits rational design of clinical trials for several reasons. Most importantly, perhaps, is the question of whether the candidate therapy targets molecular pathways independent of pirfenidone or nintedanib, and whether this can be predicted. Furthermore, without knowing which element of lung remodelling is favourably modulated by pirfenidone or nintedanib, it is challenging to predict whether a candidate medication, such as PBI-4050, will provide additional benefit to a specific cellular element of lung remodelling. This challenge is further increased by the lack of reliable preclinical models of IPF in which candidate drugs can be tested [6].

The recent publication by LUKEY et al. [15], also in this issue of the European Respiratory Journal, illustrates approaches investigators are using to overcome uncertainties when developing novel IPF therapies. Building on preclinical studies implicating the PI3K/Akt/mTOR axis in lung fibrosis [16], this phase 2 study investigated the safety, tolerability, pharmacokinetics and pharmacodynamics of the PI3K/ mTOR inhibitor omipalisib in patients with IPF. In addition to traditional pharmacokinetic measures of omipalisib in blood, the concentration of omipalisib was also measured in bronchoalveolar lavage fluid and cells harvested from lavage fluid. Target engagement was confirmed by data showing decreased pAKT/AKT and PIP3/PIP2 ratios in bronchoalveolar lavage cells. These analyses are unique because they confirmed therapeutic levels of omipalisib in the organ of interest. Not surprisingly considering the small study, there was no impact of the drug on forced vital capacity (FVC). However, a biological effect of omipalisib in lung tissue was shown by dose- and exposure-dependent reduction in fludeoxyglucose positron-emission tomography (FDG-PET) uptake in fibrotic regions of lung. Side-effects in omipalisib-treated subjects included diarrhoea and increases in insulin and glucose in some subjects. Overall, these complementary data showing that omipalisib penetrates the lung compartment, where it has a biological effect, should guide the design of larger phase 2a or phase 3 studies by informing the dose of omipalisib that optimally balances the likelihood of achieving a biological effect in the lung while limiting side-effects of the medication.

There are limitations to the approach and findings reported by LukEY et al. [15]. The design as a pharmacological and pharmacodynamic study, which generally requires a relatively small sample size to achieve significant endpoints, did not inform whether omipalisib impacts clinically meaningful endpoints such as change in FVC or survival. Further, FDG-PET uptake, the endpoint selected to confirm biological activity of omipalisib, has not been shown to be a clinically relevant pharmacodynamic endpoint in IPF. Therefore, the impact of omipalisib on FDG-PET uptake may be biologically relevant, but ultimately not prove to be clinically relevant. The study nicely defined the pharmacokinetics of omipalisib in IPF patients, but not in the context of pirfenidone or nintedanib therapy, which would have been useful when considering future clinical trial design, as shown for PBI-4050 [9]. Similarly, since omipalisib needs to be studied in the context of background therapy, it will be important to understand whether it impacts the drug levels of pirfenidone or nintedanib, potentially affecting the baseline effectiveness of combined treatment in a placebo-controlled trial.

The study reported by LUKEY et al. [15] emphasises the value of obtaining molecular data confirming a target is being engaged by novel therapeutic compounds, in the patient population of interest, prior to enrolling larger clinical trials. In a recent phase 2a study comparing the autotaxin inhibitor GLP1690, 
TABLE 1 Molecular endpoints to consider when developing novel therapies for idiopathic pulmonary fibrosis

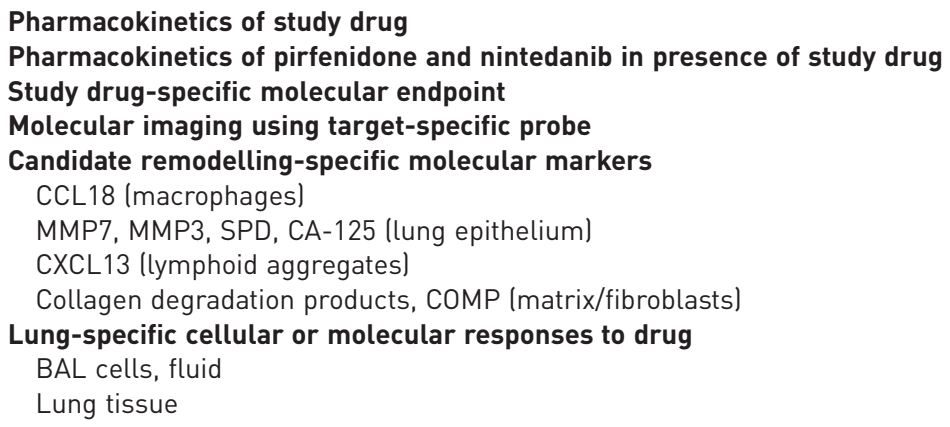

Blood/lung

Blood/lung

Blood/lung

Lung

Blood

BAL: bronchoalveolar lavage.

engagement of the target was demonstrated by assessment of serum concentration lysophosphatidic acid C18:2 in plasma [7], warranting further evaluation of safety and efficacy. Conversely, in a negative placebo-controlled study using carlumab, a monoclonal antibody against CCL2, high levels of free CCL2 were found in subjects receiving treatment, suggesting that suppression of free CCL2 for prolonged periods did not occur [17]. The data provided by LUKEy et al. [15] should be reassuring to both the sponsor and patients involved in future trials that the target is being engaged and that a study will not fail due to under-dosing the medication. Their robust evidence supporting a pharmacological effect of omipalisib, in the organ of interest, at a concentration that modulates lung fibroblasts [15], highlights an approach that could also be used to generate molecular data supporting future development of a drug for treating lung fibrosis. Additional molecular data that could be useful (table 1) are quantification of molecular markers, shown to be a readout of a molecular pathway, either in serum $[12,18]$, lavage fluid (Clinicaltrials.gov, NCT01371305), or potentially even lung tissue, provided the drug is administered to patients prior to undergoing a diagnostic lung biopsy or the molecular endpoint is quantifiable in endobronchial or transbronchial biopsy tissue. Examples include measuring changes in circulating levels of matrix degradation products, which could yield information regarding whether a drug modulates matrix turnover $[19,20]$. Similarly, CCL18, CXCL13, or MMP7, CA-125 and MMP3, could be quantified as measures of the macrophage, lymphoid aggregate or metaplastic epithelial cell element of lung remodelling in IPF patients [21-24]. Molecular imaging using probes that quantify expression of specific molecules [25] or their activity [26] are gaining traction [27] for use in drug development. These probes are attractive because they are noninvasive, specific and, theoretically, can be administered serially to individual patients to quantify relative changes in expression of a target molecule in individual patients over time.

In conclusion, the manuscript by LUKEY et al. [15] provides a framework for the type of early clinical studies and molecular endpoints to consider measuring when developing new therapies for IPF and how these endpoints may be used to establish biological activity of the drug in humans. During drug development it is also important to consider how the activity of the new treatment integrates with what is known about the pharmacological and biological activity of pirfenidone and nintedanib. Toward that end, additional molecular information is needed to define how pirfenidone and nintedanib modulate the lung remodelling and excess matrix deposition found in IPF patients, so this information can be integrated into future drug development.

Conflict of interest: P.J. Wolters reports grants and personal fees from Boehringer Ingelheim, grants from Medimmune and Genentech, and personal fees from Roche, Blade Therapeutics and Pliant, outside the submitted work. V. Cottin reports personal fees for lecturing and consultancy, and non-financial support (travel to medical meetings) from Actelion, grants, personal fees for lecturing and consultancy, and non-financial support (travel to medical meetings) from Boehringer Ingelheim and Roche, personal fees for consultancy from Bayer/MSD, personal fees for adjudication committee work from Gilead, personal fees for lecturing and consultancy from Novartis, grants from Sanofi, personal fees for data and safety monitoring board work from Promedior and Celgene, and personal fees for consultancy from Galapagos, outside the submitted work. 


\section{References}

1 Wolters PJ, Blackwell TS, Eickelberg O, et al. Time for a change: is idiopathic pulmonary fibrosis still idiopathic and only fibrotic? Lancet Respir Med 2018; 6: 154-160.

2 Wolters PJ, Collard HR, Jones KD. Pathogenesis of idiopathic pulmonary fibrosis. Annu Rev Pathol 2014; 9: $157-179$.

3 King TE Jr, Bradford WZ, Castro-Bernardini S, et al. A phase 3 trial of pirfenidone in patients with idiopathic pulmonary fibrosis. N Engl J Med 2014; 370: 2083-2092.

4 Richeldi L, du Bois RM, Raghu G, et al. Efficacy and safety of nintedanib in idiopathic pulmonary fibrosis. $N$ Engl J Med 2014; 370: 2071-2082.

5 Mora AL, Rojas M, Pardo A, et al. Emerging therapies for idiopathic pulmonary fibrosis, a progressive age-related disease. Nat Rev Drug Discov 2017; 16: 755-772.

6 Collard HR, Bradford WZ, Cottin V, et al. A new era in idiopathic pulmonary fibrosis: considerations for future clinical trials. Eur Respir J 2015; 46: 243-249.

7 Maher TM, van der Aar EM, Van de Steen O, et al. Safety, tolerability, pharmacokinetics, and pharmacodynamics of GLPG1690, a novel autotaxin inhibitor, to treat idiopathic pulmonary fibrosis (FLORA): a phase 2a randomised placebo-controlled trial. Lancet Respir Med 2018; 6: 627-635.

8 Raghu G, van den Blink B, Hamblin MJ, et al. Effect of recombinant human pentraxin 2 vs placebo on change in forced vital capacity in patients with idiopathic pulmonary fibrosis: a randomized clinical trial. JAMA 2018; 319: 2299-2307.

9 Khalil N, Manganas H, Ryerson CJ, et al. Phase 2 clinical trial of PBI-4050 in patients with idiopathic pulmonary fibrosis. Eur Respir J 2019; 53: 1800663.

10 Tzouvelekis A, Wolters PJ. Pirfenidone in the kaleidoscope: reflecting mechanisms through different angles. Eur Respir J 2018; 52: 1802046.

11 Kwapiszewska G, Gungl A, Wilhelm J, et al. Transcriptome profiling reveals the complexity of pirfenidone effects in idiopathic pulmonary fibrosis. Eur Respir J 2018; 52: 1800564.

12 Neighbors M, Cabanski CR, Ramalingam TR, et al. Prognostic and predictive biomarkers for patients with idiopathic pulmonary fibrosis treated with pirfenidone: post-hoc assessment of the CAPACITY and ASCEND trials. Lancet Respir Med 2018; 6: 615-626.

13 Zhang Y, Jones KD, Achtar-Zadeh N, et al. Histopathological and molecular analysis of idiopathic pulmonary fibrosis lungs from patients treated with pirfenidone or nintedanib. Histopathology 2019; 74: 341-349.

14 Wuyts WA, Antoniou KM, Borensztajn K, et al. Combination therapy: the future of management for idiopathic pulmonary fibrosis? Lancet Respir Med 2014; 2: 933-942.

15 Lukey PT, Harrison SA, Yang S, et al. A randomised, placebo-controlled study of omipalisib (PI3K/mTOR) in idiopathic pulmonary fibrosis. Eur Respir J 2019; 53: 1801992.

16 Mercer PF, Woodcock HV, Eley JD, et al. Exploration of a potent PI3 kinase/mTOR inhibitor as a novel anti-fibrotic agent in IPF. Thorax 2016; 71: 701-711.

17 Raghu G, Martinez FJ, Brown KK, et al. CC-chemokine ligand 2 inhibition in idiopathic pulmonary fibrosis: a phase 2 trial of carlumab. Eur Respir J 2015; 46: 1740-1750.

18 Jia G, Chandriani S, Abbas AR, et al. CXCL14 is a candidate biomarker for Hedgehog signalling in idiopathic pulmonary fibrosis. Thorax 2017; 72: 780-787.

19 Jenkins RG, Simpson JK, Saini G, et al. Longitudinal change in collagen degradation biomarkers in idiopathic pulmonary fibrosis: an analysis from the prospective, multicentre PROFILE study. Lancet Respir Med 2015; 3: 462-472.

20 Maher TM, Stowasser S, Nishioka Y, et al. Investigating the effects of nintedanib on biomarkers of extracellular matrix turnover in patients with IPF: design of the randomised placebo-controlled INMARK(R)trial. BMJ Open Respir Res 2018; 5: e000325.

21 DePianto DJ, Chandriani S, Abbas AR, et al. Heterogeneous gene expression signatures correspond to distinct lung pathologies and biomarkers of disease severity in idiopathic pulmonary fibrosis. Thorax 2015; 70: 48-56.

22 Maher TM, Oballa E, Simpson JK, et al. An epithelial biomarker signature for idiopathic pulmonary fibrosis: an analysis from the multicentre PROFILE cohort study. Lancet Respir Med 2017; 5: 946-955.

23 Prasse A, Pechkovsky DV, Toews GB, et al. CCL18 as an indicator of pulmonary fibrotic activity in idiopathic interstitial pneumonias and systemic sclerosis. Arthritis Rheum 2007; 56: 1685-1693.

24 Rosas IO, Richards TJ, Konishi K, et al. MMP1 and MMP7 as potential peripheral blood biomarkers in idiopathic pulmonary fibrosis. PLoS Med 2008; 5: e93.

25 John AE, Luckett JC, Tatler AL, et al. Preclinical SPECT/CT imaging of alphavbeta6 integrins for molecular stratification of idiopathic pulmonary fibrosis. J Nucl Med 2013; 54: 2146-2152.

26 Withana NP, Ma X, McGuire HM, et al. Non-invasive imaging of idiopathic pulmonary fibrosis using cathepsin protease probes. Sci Rep 2016; 6: 19755.

27 Montesi SB, Desogere P, Fuchs BC, et al. Molecular imaging of fibrosis: recent advances and future directions. J Clin Invest 2019; 129: 24-33. 